\title{
Development of a shock-induced detonation driver
}

\author{
F.K. Lu and D.R. Wilson
}

Aerodynamics Research Center, University of Texas at Arlington, Arlington, TX 76019, USA

\section{Introduction}

A shock tube's performance can be improved by using a detonation driver that produces a driver gas with a high pressure, a high temperature, a low specific heat ratio and a high speed of sound. Major drawbacks, however, are that the gaseous detonation products have high molecular weight compared to helium and the potential danger. The detonation driver technique, while not new, has been re-examined lately [1,2].

\section{Facility development and description}

Details of the detonation-driven shock tube shown schematically in Fig. 1a can be found in [3]. The driver and detonation sections, rated at 408 atm and separated by a double diaphragm section, have bores of $152.4 \mathrm{~mm}$ and lengths of 3.05 and $2.74 \mathrm{~m}$ respectively. The detonation and driven sections are separated by a mylar diaphragm. The driven tube, rated at $188 \mathrm{~atm}$, is $9 \mathrm{~m}$ long and has a $40 \mathrm{~mm}$ bore. A $471 \mathrm{~mm}$ long test section is located $3.16 \mathrm{~m}$ from the primary diaphragm. A $6.12 \mathrm{~m}$ long tube installed downstream of the test section prevents interference of the test flow from wave reflections from the open end. A mylar diaphragm $3.07 \mathrm{~m}$ downstream of the test section contains the initial driven tube gas. Data are recorded simultaneously at $100 \mathrm{kHz} /$ channel. The TEP ${ }^{1}$ code is used to estimate the facility performance, assuming a quasi-1D equilibrium flow.

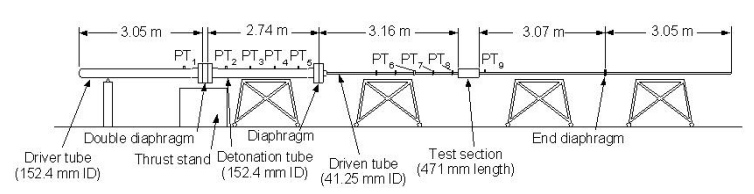

a. Schematic: PT = flush-mounted pressure transducers; 1 is located in the driver tube; $2-5=0.495,1.08,1.66$ and $2.25 \mathrm{~m}$ upstream of the mylar diaphragm separating the detonation and driven tubes; $6-9$ $=2.146,2.604,3.061$ and $3.416 \mathrm{~m}$ downstream

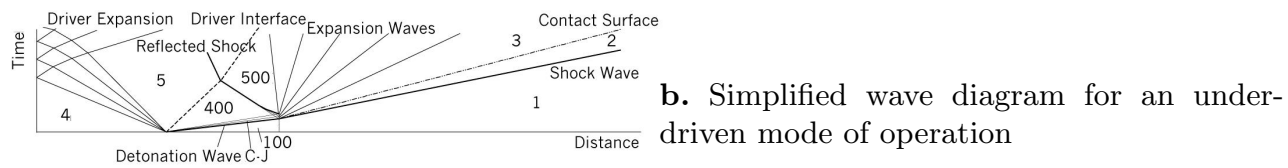

Fig. 1. Shock-induced detonation driven shock tube

The high-pressure driver gas expanding into the detonation section propagates a shock wave, transitioning into a detonation wave in an estimated length of $0.2 \mathrm{~m}$ (Fig. 1b). The propagating detonation wave impinges and ruptures the thin downstream diaphragm. The area reduction causes partial wave reflection, trapping high-pressure gas as in a reflected shock tunnel. This alleviates attenuation from the Taylor rarefaction, prolonging the uniform flow duration within the driven tube. After a successful run, the end diaphragm is blown away and the entire shock tube is vented.

\footnotetext{
1 Thermodynamic Equilibrium Program, a Windows ${ }^{\mathrm{TM}}$ version of the NASA CEA (Chemical Equilibrium with Applications) code (http://www.grc.nasa.gov/WWW/CEAWeb/)
} 
Gas momentum yields extra performance when the detonation driver is operated in the downstream mode [1,2]. High-pressure air or helium driver gas, when released by diaphragm rupture, initiates a detonation wave propagating to the right in the detonation tube filled with stoichiometric oxyhydrogen. This detonation wave induces the test flow in the driven tube. A quasi-uniform flow 2 exists behind the shock, to be followed by an expansion 3-500. The Taylor rarefaction between 100 and 400, following the detonation wave, can cause a non-uniform "pumping" in the driven tube and resulting in an "underdriven" mode of operation. The shock driver in some cases can eliminate the rarefaction to produce a "perfectly driven" mode [1]. An "over-driven" mode is also possible.

\section{Results and discussion}

Detonation tube pressures for an under-driven mode of operation are shown in Fig. 2. The first peak is due to the incident detonation wave and the second to the wave reflected from the downstream end of the detonation tube. With $u_{C J}=a_{C J}$, TEP yields the CJ parameters and the state of the detonation products. Incomplete fuel-oxidizer mixing is thought to cause the experimental value of $u_{d}$ to differ from the theoretical $u_{C J}[3]$. But, if the difference between the measured and the CJ velocities is within $10 \%$, detonation is assumed to be achieved since this criterion clearly distinguishes the detonation wave from a propagating shock. The calculated CJ parameters using TEP are corrected for incomplete mixing. An appropriate portion of fuel which produces the measured velocity is substituted with an equivalent amount of a nonreactive gas of the same molecular weight, such as a $\mathrm{He}$ atom for a $\mathrm{H}_{2}$ molecule. The corrected $p_{C J}$ is shown in Fig. 2 . It can be seen that the pressure transducer is unable to resolve the theoretical $p_{C J}$.

Assuming isentropic expansion and a calorically perfect gas,

$$
a_{5} / a_{4}=\left(p_{5} / p_{4}\right)^{\left[0.5\left(\gamma_{4}-1\right) / \gamma_{4}\right]}
$$

whereby, from the characteristic curve between regions 4 and 5 ,

$$
\begin{aligned}
& p_{4} / p_{5}=\left[1+0.5\left(\gamma_{4}-1\right) u_{5} / a_{5}\right]^{\left[2 \gamma_{4} /\left(\gamma_{4}-1\right)\right]} \\
& \left(p_{5} / p_{4}\right)^{\left[\left(\gamma_{4}-1\right) / 2 \gamma_{4}\right]}=1-0.5\left(\gamma_{4}-1\right) u_{5} / a_{4}
\end{aligned}
$$

In the Taylor rarefaction that follows the detonation wave, the same characteristics give

$$
\left(p_{400} / p_{C J}\right)^{\left[\left(\gamma_{C J}-1\right) / 2 \gamma_{C J}\right]}=1-0.5\left(\gamma_{C J}-1\right)\left(u_{C J}-u_{400}\right) / a_{C J}
$$

Now, $p_{5}=p_{400}$ and $u_{5}=u_{400}$. Thus, from (2) and (3),

$$
p_{400}^{f(4)}+A p_{400}^{f(C J)}+B=0
$$

where $A=\left(\gamma_{4}-1\right) /\left(\gamma_{C J}-1\right)\left(a_{C J} / a_{4}\right) p_{C J}^{-f(C J)} p_{4}^{f(4)}, \quad f(i)=0.5\left(\gamma_{i}-1\right) / \gamma_{i}$, $B=\left[0.5\left(\gamma_{4}-1\right) u_{C J} / a_{4}-\left(\gamma_{4}-1\right) /\left(\gamma_{C J}-1\right) a_{C J} / a_{4}-1\right] p_{4}^{f(4)}$. With the CJ and initial driver conditions, the plateau pressure of region 400 can be obtained numerically using (4). The pressure $p_{400}$ calculated by (4) is indicated in Fig. 2 and listed in Table 1. Although it is hard to uniquely determine the experimental plateau pressure, Fig. 2 shows a good agreement with the theoretical value.

From (3), $u_{400}=u_{5}$, the speed at the head of the expansion in the high-pressure driver, is also calculated. Therefore, the driver interface arrival at $\mathrm{PT}_{4}$ and $\mathrm{PT}_{5}$ are calculated as 3.61 and $4.88 \mathrm{~ms}$, respectively. A slight modification of (2) yields

$$
a_{400}=0.5\left(\gamma_{4}-1\right) u_{400} /\left[1-\left(p_{400} / p_{4}\right)^{\left[0.5\left(\gamma_{4}-1\right) / \gamma_{4}\right]}\right]
$$


The tail of the Taylor rarefaction advances at a speed of $\left(a_{400}+u_{400}\right)$ resulting in its calculated arrival at $\mathrm{PT}_{4}$ and $\mathrm{PT}_{5}$ to be 0.89 and $1.21 \mathrm{~ms}$. In general, the measured detonation wave speeds in the detonation tube are within $10 \%$ of $u_{C J}$. A possible reason for the lower measured values in the driven tube is boundary-layer growth [2].

Table 1. Example of initial conditions and calculated properties for the example of Fig. 2

\begin{tabular}{|c|c|c|c|c|c|c|}
\hline \multirow{2}{*}{ Tube } & \multicolumn{3}{|c|}{ Initial Conditions } & \multicolumn{3}{|c|}{ Calculated Properties } \\
\hline & Gas & Region & $p$, atm & Region & $p$, atm & $T, \mathrm{~K}$ \\
\hline Driver & Air & 4 & 183 & 5 & 20.9 & 161 \\
\hline \multirow{3}{*}{ Detonation } & \multirow{3}{*}{$2 \mathrm{H}_{2}+\mathrm{O}_{2}$} & \multirow{3}{*}{100} & \multirow{3}{*}{2.01} & CJ & 37.7 & 3630 \\
\hline & & & & 400 & 20.9 & 3390 \\
\hline & & & & 500 & 37.1 & 3790 \\
\hline Driven & Air & 1 & 0.97 & 2 & 14.4 & 975 \\
\hline
\end{tabular}
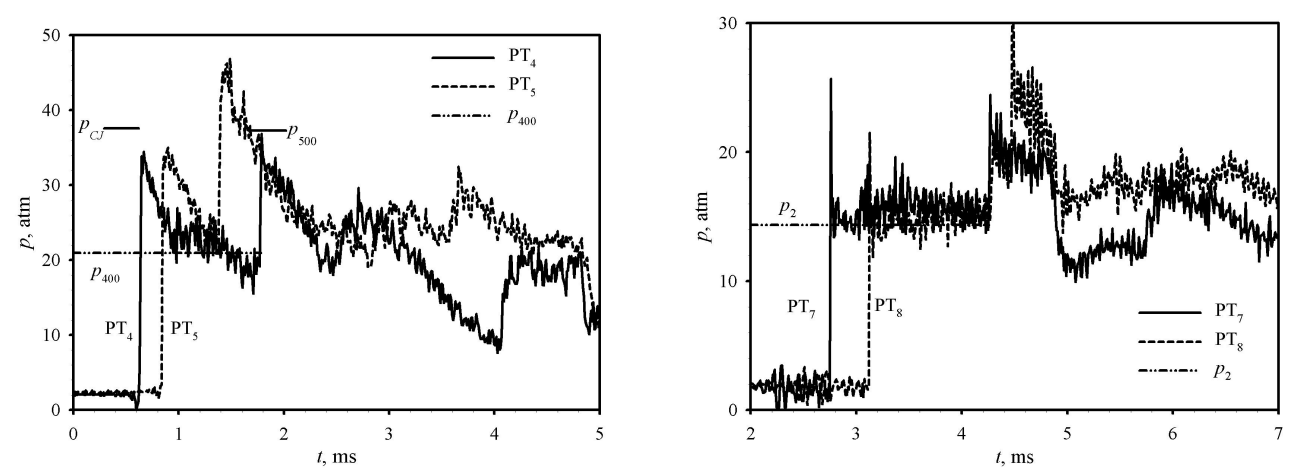

Fig. 2. Detonation tube pressures for a stoi- Fig. 3. Pressures in driven tube for case of chiometric oxyhydrogen mixture initially at 2 Fig. 2

atm, with an air driver $\left(p_{4}=219 \mathrm{~atm}, T_{4}=300 \mathrm{~K}\right)$; abscissa is the time delay after the estimated instant of diaphragm breakage

\subsection{Reflected and transmitted shocks}

The plateau pressure $p_{400}\left(=p_{5}\right)$ and the reflected shock speed govern the state of 500 . TEP values of $p_{500}$ and $T_{500}$ for the example of Fig. 2 are listed in Table 1 . The TEP value of $p_{500}$ agrees well with the measured peak pressure (Fig. 2). The peak pressure measured at $\mathrm{PT}_{5}$ is higher than that at $\mathrm{PT}_{4}$. The reason for this is that when the detonation wave arrives at the mylar diaphragm, the pressure behind the detonation wave is higher than $p_{400}$ since it is not yet attenuated by the Taylor rarefaction. As the detonation wave reflects and propagates through the gas at higher pressure, the post-shock pressure becomes temporarily higher than $p_{500}$. The reflected shock accelerates under progressive attenuation by the Taylor rarefaction until it encounters the plateau pressure. The gas velocity after the reflected shock $u_{500}$ is calculated to be $-277 \mathrm{~m} / \mathrm{s}$. The downstream propagating detonation wave is partially transmitted into the driven tube. Pressures recorded in the driven tube are shown in Fig. 3.

\subsection{Effect of reactants}

Figure 4 summarizes the detonation velocity for different stoichiometric reactant pairs. The experimental velocities are generally lower than the calculated values except for 


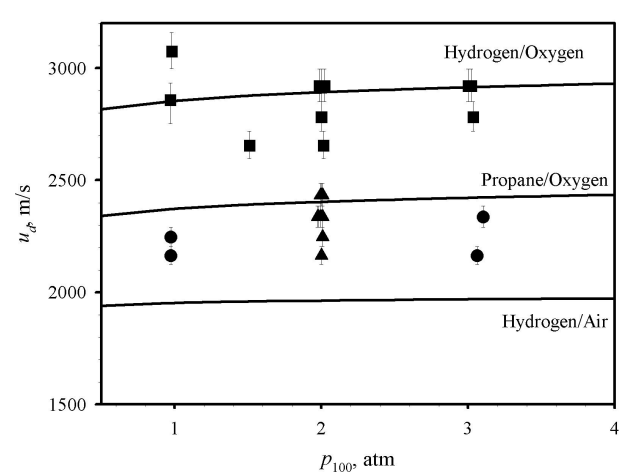

Fig. 4. Detonation velocity dependence on reactants (stoichiometric mixtures in all cases at initial pressures of $0.5-4 \mathrm{~atm})$ : TEP results are shown as lines, experimental values for oxyhydrogen/oxygen are shown as squares, for propane/oxygen as triangles, and for hydrogen/air as circles (vertical error bars are due to the $10 \mu$ s temporal resolution)

the hydrogen/air cases, which are as much as $20 \%$ higher. For hydrogen/air, incomplete mixing may produce oxyhydrogen mixtures locally, resulting in the higher velocity.

Figure 5 compares the measured and calculated post-detonation pressure. The value of $p_{C J}$ is difficult to determine experimentally because of the rapid initial decay of pressure from its peak, von Neumann value. To compare the post-detonation pressure, the pressure is averaged over $0.1 \mathrm{~ms}$ after the peak to provide consistency in its evaluation. Thus, $\bar{p}_{C J}$ is the averaged pressure assuming that it represents $p_{C J}$. The experiments and calculations both indicate that the highest pressures are obtained with propane/oxygen mixtures. The hydrogen/air cases show that most departures from the theoretical values and are likely due to incomplete mixing.

\subsection{Driver gas}

The uniform region 400 of detonation products is determined by the state of the driver gas in region 4 and CJ parameters as indicated by (4). The theoretical uniform pressures when an air, helium or hydrogen driver is used to detonate a stoichiometric oxyhydrogen or hydrogen/air mixture initially at $2 \mathrm{~atm}$ and $300 \mathrm{~K}$ are shown in Fig. 6. Note that while the figure also includes TEP results for a hydrogen driver, this approach is not used in the experiments.

For a stoichiometric oxyhydrogen mixture, strong Taylor rarefaction prevents $p_{400}$ from reaching $p_{C J}$ with an air driver for the $p_{4}$ range. Only an under-driven mode is achieved. For hydrogen driving a stoichiometric oxyhydrogen mixture, the pressure of the hydrogen driver just balances the pressure at the rear of the detonation wave $p_{C J}$ at $185 \mathrm{~atm}$, annihilating the Taylor rarefaction. In this perfectly driven mode, the full CJ pressure level can ideally be maintained behind the detonation wave. A further increase in $p_{4}$ causes the expanded driver gas pressure to be higher than the CJ value, yielding an "over-driven" condition where $u_{d}>u_{C J}$. Since hydrogen is not used as a driver gas, none of the reported experiments achieved over-driven conditions and very few achieved nearly perfectly-driven conditions for the range of fill pressures attempted. The experimental 
plateau pressure $\bar{p}_{400}$ is obtained approximately by averaging over $0.25 \mathrm{~ms}$ before the reflected shock arrives at $\mathrm{PT}_{4}$. The experimental values, plotted in Fig. 6, are lower than theoretical, implying that the Taylor rarefaction is stronger than theoretical predictions.

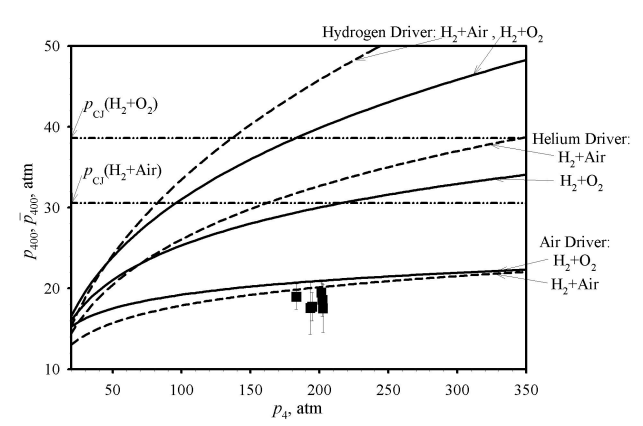

Fig. 6. Plateau pressures for air, helium, and hydrogen drivers (stoichiometric oxyhydrogen or hydrogen/air mixtures at $2 \mathrm{~atm}$ and $300 \mathrm{~K}$ initial conditions, solid and dashed lines respectively); CJ pressures are shown as chain lines

Figure 7 shows nearly perfectly-driven pressure traces. The theoretical and experimental $p_{C J}$ and $p_{400}$ agree well with each other. The reflected shock here produces a higher pressure than with an air driver. After the reflected shock, the pressure even increases due to blowdown of the high-pressure, driver tube gas. The helium driver interface advances more than twice as fast as the air driver interface; $u_{5}=410$ and $860 \mathrm{~m} / \mathrm{s}$ for the air and helium drivers respectively. The location where the driver interface meets the reflected shock is estimated to be $0.29 \mathrm{~m}$ upstream of $\mathrm{PT}_{5}$, which means that the reflected shock measured at $\mathrm{PT}_{4}$ is propagating not through the detonated gas but through the helium expanded from the driver. In this case, $p_{500}$ calculated using the previous method is no longer valid since the reflected shock speed is reduced as it encounters the helium at a lower temperature, that is, $a_{5}<a_{400}$, causing $p_{500}$ to be underestimated in Fig. 7 .

\subsection{Driven tube conditions}

Figure 8a shows an example of detonation tube pressure histories for an under-driven condition. The detonation tube contains a stoichiometric oxyhydrogen mixture at an initial pressure of $1.5 \mathrm{~atm}$, and the driver tube is pressurized to $219 \mathrm{~atm}$ with air. TOF results indicate a detonation wave propagation speed of $2920 \mathrm{~m} / \mathrm{s}$, which is almost identical to the theoretical CJ detonation wave speed for these conditions. For this under-driven mode, the incident detonation wave is followed by a Taylor rarefaction that lowered the pressure. The reflected detonation wave increases the pressure by a factor of 2.3 , which agrees with CJ theory.

Figure $8 \mathrm{~b}$ shows an example of a nearly perfectly-driven mode. The rate of pressure drop in this case is about the same as previously. But, the absolute pressure level achieved by the reflected detonation wave is much higher. This increase in reflected pressure increases the driven-tube Mach number from 6.70 to 7.65. Figure 9 shows the corresponding pressure histories in the driven tube. The duration of quasi-uniform flow is at least $1 \mathrm{~ms}$. The contact surface follows the shock wave after $0.76-0.79 \mathrm{~ms}$. For a shock speed of $\mathcal{O}(300-400) \mathrm{m} / \mathrm{s}$, this yields a $220-300 \mathrm{~mm}$ long slug of test gas. 
$\mathbf{a}$

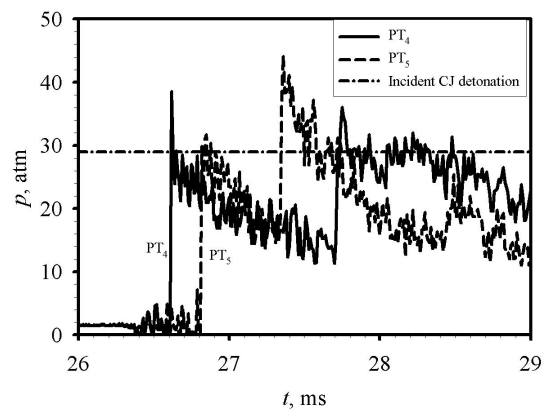

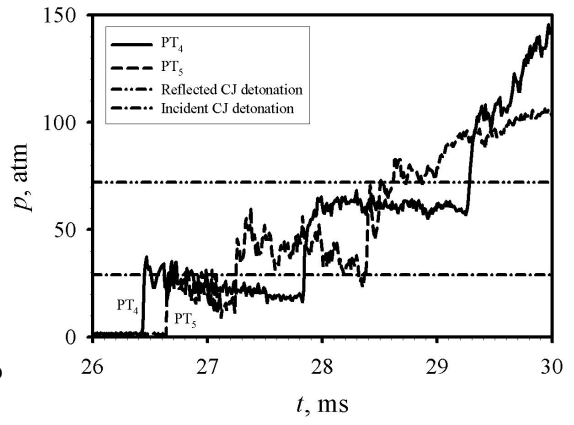

Fig. 8. Detonation tube pressure traces for a stoichiometric oxyhydrogen mixture at an initial pressure of $1.5 \mathrm{~atm}$ with shock-induced detonation: a air driver (initial conditions of $219 \mathrm{~atm}$ and $300 \mathrm{~K}$ ), b helium driver (initial conditions of $201 \mathrm{~atm}$ and $300 \mathrm{~K}$ )

a

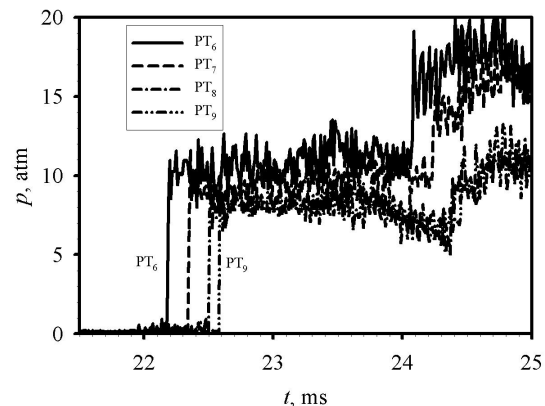

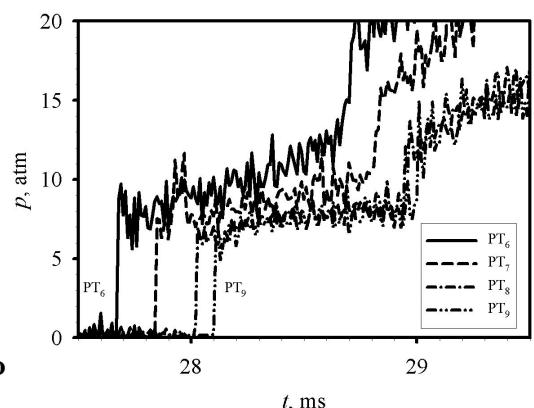

Fig. 9. Driven tube pressure traces corresponding to Figs. 8a and b

\section{Conclusions}

A driver tube mitigated the effect of the Taylor rarefaction in a detonation-driven shock tube operated in the forward mode to yield an adequate duration of uniform, highpressure test flow. The shock-induced detonation tube may approach the perfectly-driven mode when a light gas is used as the driver gas, the initial driver pressure and temperature are high, the post-detonation pressure is low, and $a_{C J}$ and $u_{C J}$ are small.

Acknowledgement: The facility development was partly funded by MSE, Inc., Butte, Montana and by the Texas Advanced Technology Program (Grants 003656-056 \& 003656-031).

\section{References}

1. F.K. Lu, D.R. Wilson, R.J. Bakos, J.I. Erdos: Recent advances in detonation techniques for high-enthalpy facilities. AIAA J 38(9), 1676 (2000)

2. H. Olivier, Z. Jiang, H. Yu, F.K. Lu: 'Detonation driven shock tubes and tunnels'. In: Advanced hypersonic facilities. AIAA, Reston, Virginia, 2002, ed. by F.K. Lu, D.E. Marren (2002) pp.135-203

3. F.K. Lu, D.R. Wilson: Detonation driver for enhancing shock tube performance. Shock Waves, (2003), (in press) 